\title{
Overexpression of caveolin-1 reduces Taxol resistance in human osteosarcoma cells by attenuating PI3K-Akt-JNK dependent autophagy
}

\author{
JIAN GUAN*, ZHENCHAO YUAN*, JULIANG HE, ZHENJIE WU, BIN LIU, XIANG LIN, LIGEN MO and HAO MO \\ Department of Bone and Soft Tissue Neurosurgery, Affiliated Tumor Hospital of Guangxi Medical University, \\ Nanning, Guangxi 530021, P.R. China
}

Received March 27, 2016; Accepted May 11, 2016

DOI: $10.3892 /$ etm.2016.3713

\begin{abstract}
Caveolin-1 (CAV-1), which is an oncoprotein and a tumor suppressor, is highly expressed in normal osteoblasts. Although researchers have investigated its role in human osteosarcoma, the mechanism of caveolin-1 action in osteosarcoma remains unknown. In the present study, Saos-2 and U-2 OS cells were cultured with a continuous induction protocol of gradually increasing Taxol concentration for 6 months to establish drug-resistant cell lines. CAV-1 expression levels in osteosarcoma cells were detected via western blotting and quantitative polymerase chain reaction. CAV-1 knockdown was achieved using a short hair-pin RNA lentivirus vector, and cell viability was analyzed by MTT assay. The effect of caveolin-1 on autophagy was investigated, and the downregulation of caveolin-1 and increased autophagy was identified in Taxol-resistant osteosarcoma cells. In addition, the results of the present study demonstrated that downregulation of caveolin-1 promotes autophagy and induces osteosarcoma cell resistance to Taxol. Notably, overexpression of CAV-1 resensitized drug-resistant cells to Taxol via declined autophagy. In conclusion, CAV-1 was demonstrated to be downregulated in Taxol-resistant osteosarcoma cells, and overexpression of CAV-1 in human osteosarcoma cells suppressed Taxol resistance by attenuating PI3K-Akt-JNK-dependent autophagy. The present findings suggest that further investigation into CAV-1's role in Taxol resistance is warranted. In the future, detection of CAV-1 may be used as an indicator to evaluate the treatment and prognosis of patients with osteosarcoma.
\end{abstract}

Correspondence to: Dr Hao Mo, Department of Bone and Soft Tissue Neurosurgery, Affiliated Tumor Hospital of Guangxi Medical University, 71 He Di Road, Nanning, Guangxi 530021, P.R. China

E-mail: mohaogxzlyy@sina.com

*Contributed equally

Key words: caveolin-1, Taxol resistance, osteosarcoma cell, Janus kinase, autophagy

\section{Introduction}

Osteosarcoma is the most prevalent malignant primary sarcoma of the bone in children and adolescents, and is a leading cause of cancer-associated mortality in young adults, accounting for $3-4 \%$ of all malignancies and $30 \%$ of malignant bone tumors in adolescents (1). The development of effective chemotherapeutic treatment has improved five-year survival rates of patients with localized osteosarcoma in the US, from 20 to between 65 and $70 \%$ and the success of limb-sparing surgery over the past several decades (1); however, individuals with metastatic osteosarcoma continue to exhibit poor prognoses, with long-term survival rates only observed in $<20 \%$ of patients. Despite the advancement of chemotherapeutic treatment, negative side effects, metastasis, disease recurrence and drug resistance have perpetuated poor outcomes for patients. Therefore, exploiting novel treatment strategies is crucial for osteosarcoma therapy.

The autophagy pathway has a critical role in maintaining cellular homeostasis by delivering macromolecules and organelles from the cytoplasm to lysosomes for degradation $(2,3)$. Various stress conditions, including energy deprivation, nutrient starvation and oxidative stress, may rapidly induce autophagy, which has a critical role in maintaining cell homeostasis and survival (4). However, consistent and prolonged activity of autophagy may induce autophagic cell death. Dysregulation of autophagy has been observed in various disease states, including infectious disease, cancer and neurodegenerative diseases $(4,5)$.

The role of autophagy in cancer is complicated, and a previous study has referred to it as a 'double-edged sword' (2). In particular, it has been demonstrated that autophagy suppresses mammary tumorigenesis driven by WNT1 activation (6); however, there is also evidence to suggest that autophagy is an important mechanism for cancer cell survival (7). Therefore, whether autophagy inhibits or propagates cancer may depend on the type of cancer, therapeutic strategy, or both. A comprehensive understanding of all the autophagy factors is required to predict whether autophagy will protect cancer cells or kill them.

Drug resistance is a primary cause of cancer treatment failure. Many strategies to overcome drug resistance in cancer have been studied. For example, Wang et al (8) have previously reported that HMGB1-mediated autophagy promotes neuroblastoma cell chemoresistance; protective autophagy has been 
demonstrated to promote lapatinib resistance in HER2-positive breast cancer cells (9); Giuliano et al (10) have demonstrated that inhibition of autophagy leads to sunitinib resistance in renal clear cell carcinoma; and in a study conducted by Crystal et al (11), MEK activation was revealed to promote ceritinib resistance and MEK inhibitor treatment was able to reverse resistance to ceritinib. A recent study indicated that caveolin-1 (CAV-1) was highly expressed in cancer stem cells and decreased cells' chemosensitivity (12). CAV-1 inhibition has previously been shown to be associated with autophagic induction in human breast cancer cells (13). Furthermore, it has been demonstrated that CAV-1 deletion increases basal autophagy, due to an increase in the complex of autophagy-related proteins 5 and 12 (Atg5-Atg12), and that a CAV-1 binding motif mutation broke this complex and accelerated autophagy (14). In addition, previous studies have reported that CAV-1 deficiency was an independent factor for the poor prognosis of colorectal cancer, demonstrating that loss of CAV-1 may increase drug resistance and cancer metastasis $(15,16)$.

In present study, Saos-2 and U-2 OS cells were cultured with gradually increasing concentrations of Taxol, in order to establish drug-resistant cell lines. The findings of the present study suggest that further investigation into the association between CAV-1 and Taxol resistance is warranted.

\section{Materials and methods}

Cell culture and lentivirus infection. Human osteosarcoma cell lines were purchased from American Type Culture Collection (Manassas, VA, USA). Saos-2/Taxol and U-2 OS/Taxol cells were established via gradually increasing the concentration of Taxol, every fortnight $(5,10,20,50$, $100,150,200,250$, and $300 \mathrm{ng} / \mathrm{ml}$ ). DNA oligonucleotides carrying small hairpin (sh) RNA (Invitrogen; Thermo Fisher Scientific, Inc., Waltham, MA, USA) were constructed into pLKO.1 plasmids (Addgene; Cambridge, MA, USA). Packaging (psPAX2) and envelope (pMD2.G) plasmids (Addgene, Inc) were transfected into HEK293T cells with recombinant plasmids. The supernatant containing lentiviruses were collected after $36 \mathrm{~h}$. The short hairpin (sh)RNA used to assess caveolin-1 (CAV-1) and autophagy related protein 5 (Atg5) were as follows: i) shCAV-1\#1, CATCTACAAGCCCAA CAAC; ii) shCAV-1\#2, AGACGAGCTGAGCGAGAAG; iii) shAtg5\#1, ATTGGCTCAATTCCATGAA; iv) shAtg5\#2, GCTACTCTGGATGGGATTG; and v) control shRNA, CACACCGTTTCGTGGCTTT. The following inhibitory compounds (all $10 \mu \mathrm{M}$ in culture medium) were used to treat cells in the present study: i) Bafilomycin A1 (autophagy inhibitor) for $4 \mathrm{~h}$ (Baf A1; cat. no. ALX-380-063-M001; Enzo Life Sciences, Inc., Farmingdale, NY, USA); ii) MK-2206 (Akt inhibitor) for $1 \mathrm{~h}$ (cat. no. 1888-500; BioVision, Inc., Milpitas, CA, USA); iii) SP600125 (JNK inhibitor) for $1 \mathrm{~h}$ (cat. no. S5567; Sigma-Aldrich, St. Louis, MO, USA); and iv) LY294002 (PI3K inhibitor) for $1 \mathrm{~h}$ (cat. no. L9908; Sigma-Aldrich).

Cell viability assay. Cell viability was analyzed via MTT assay using a Roche Cell Proliferation Kit I (Roche Diagnostics, Basel, Switzerland; cat. no. 11465007001) according to the manufacturer's protocol. All experiments were performed in triplicate. Results were plotted using Prism5 software (GraphPad Software, Inc., La Jolla, CA, USA).

Reverse transcription-quantitative polymerase chain reaction (RT-qPCR) analysis. Total RNA from osteosarcoma cells was extracted using TRIzol reagent (Invitrogen; Thermo Fisher Scientific, Inc.) and $4 \mu \mathrm{g}$ was used for reverse transcription (RT). RT was performed using a first-strand cDNA synthesis kit (Thermo Fisher Scientific, Inc.), according to the manufacturer's protocol. qPCR analysis was carried out using a SYBR Green kit (TransGen Biotech Co. Ltd., Beijing, China) on an ABI 7900 system (Applied Biosystems; Thermo Fisher Scientific, Inc.). qPCR reactions (20 $\mu$ l total volume) comprised the following: SYBR, $10 \mu \mathrm{l}$; cDNA, $1 \mu \mathrm{l}$; forward primer, $0.25 \mu \mathrm{l}$; reverse primer, $0.25 \mu \mathrm{l}$; ROX reference dye, $0.1 \mu \mathrm{l}$; and double-distilled $\mathrm{H}_{2} \mathrm{O}, 8.4 \mu \mathrm{l}$. Primers were designed as follows: CAV-1, forward 5'-AACACGTAGCTGCCCTTCAG-3' and reverse 5'-GGATGGGAACGGTGTAGAGAT-3'; and ACTB, forward 5'-TGTTTGAGACCTTCAACACCC-3' and reverse 5'-AGCACTGTGTTGGCGTACAG-3'. The amplification conditions were as follows: Pre-denaturation at $94^{\circ} \mathrm{C}$ for $5 \mathrm{~min}, 40$ cycles of denaturation at $94^{\circ} \mathrm{C}$ for $30 \mathrm{sec}$, annealing at $59^{\circ} \mathrm{C}$ for $30 \mathrm{sec}$, extension at $72^{\circ} \mathrm{C}$ for $25 \mathrm{sec}$ and a final extension at $72^{\circ} \mathrm{C}$ for $10 \mathrm{~min} . \mathrm{Cq}$ values were measured during the exponential amplification phase. Relative gene expression was calculated using the $2^{-\Delta \Delta \mathrm{Cq}}$ method (17), with ACTB as the internal control gene. For each gene RT-qPCR was performed in triplicate.

Western blot analysis. Cells were lysed in RIPA buffer (Sigma-Aldrich). The concentration of the protein samples was determined by the Bradford method (18). Lysates were denatured at $100^{\circ} \mathrm{C}$ for $10 \mathrm{~min}$ and subsequently cooled on ice. A total of $40 \mu \mathrm{g}$ protein was separated by $8-15 \%$ SDS-PAGE and transferred onto a polyvinylidene difluoride membrane (GE Healthcare Life Sciences, Chalfont, UK). The primary antibodies used in the present study were: i) $\beta$-actin (cat. no. A1978; Sigma-Aldrich; mouse; monoclonal; 1:5,000 in 5\% w/v milk); ii) microtubule associated protein 1 light chain 3 [(LC3; cat. no. 2775; rabbit polyclonal; 1:5,000 in 5\% w/v bovine serum albumin (BSA; Sangon Biotech Co., Ltd., Shanghai, China); iii) Atg5 (cat. no. 2630; rabbit polyclonal, 1:2,000 in $5 \%$ w/v BSA); iv) phosphorylated janus kinase (p-JNK; T183/ Y185; cat. no. 4668; rabbit; polyclonal; 1:1,000 in 5\% w/v BSA); v) JNK (cat. no. 9252; rabbit; polyclonal; 1:2,000 in 5\% w/v BSA); vi) CAV-1 (cat. no. 3238; rabbit; polyclonal; 1:2,000 in 5\% w/v BSA); vii) Akt (cat. no. 9272; rabbit; polyclonal; 1:2,000 in 5\% w/v BSA); viii) p-Akt (S473; cat. no. 4060; rabbit; polyclonal; 1:2,000 in 5\% w/v BSA); ix) Atg7 (cat. no. 2631; rabbit; polyclonal; 1:2,000 in 5\% w/v BSA); and x) Beclin1 (cat. no. 3495; rabbit; polyclonal; 1:2,000 in 5\% w/v BSA) (all Cell Signaling Technologies, Inc., Danvers, MA, USA).

Laser scanning confocal microscopy. Cells stably expressing ref fluorescent protein (RFP)-LC3 were cultured in live cell imaging culture dishes (Livefocus, Jiangsu, China; cat. no. C-L-8) and subjected to serum starvation in Hank's balanced salt solution (Thermo Fisher Scientific, Inc.) or lentivirus. Living cells were visualized using a Zeiss LSM510 
meta-confocal system (Zeiss AG, Oberkochen, Germany). A total of 200 cells were detected at each condition, and significant differences were analyzed by Student's t-test.

Statistical analysis. Statistical analyses were conducted using GraphPad Prism5 (GraphPad Software, Inc.). Significant differences were analyzed by performing a Student's t-test. $\mathrm{P}<0.05$ was considered to indicate a statistically significant difference.

\section{Results}

CAV-1 expression is reduced in Taxol-resistant human osteosarcoma cells. A recent study indicated that CAV-1 was highly expressed in cancer stem cells and decreased cells' chemosensitivity (12). To explore the expression levels of caveolin-1 in Taxol-resistant osteosarcoma cells, a qPCR assay was performed to detect CAV-1 mRNA expression levels (Fig. 1A). The results indicated that CAV-1 expression levels were significantly decreased in Taxol-resistant human osteosarcoma cells, compared with their non-resistant counterparts $(\mathrm{P}<0.05$; Fig. 1A). Western blot analysis was performed to examine the protein expression levels of CAV-1 in Saos-2/Taxol, U-2 OS/Taxol and their drug-sensitive parent cells (Fig. 1B). These findings were consistent with the results of qPCR analysis. Caveolin-1 expression was markedly reduced in Taxol-resistant human osteosarcoma cells, as compared with the Soas- 2 and U-2 OS controls.

Basal autophagy is higher in Saos-2/Taxol and U-2 OS/Taxol cells, compared with Saos-2 and U-2 OS cells. CAV-1 inhibition has previously been shown to be correlated with autophagic induction in human breast cancer cells (13). Autophagy is a regulatory mechanism that protects cells from stress; however, prolonged consistent autophagy may cause type II programmed cell death (4). To detect autophagy in the present study, canonical markers of autophagy in Saos-2/Taxol, U-2 OS/Taxol and their drug sensitive parents cells were analyzed (Fig. 1B). Atg5, Atg7, LC3 II and Beclin1 were demonstrated to be highly expressed in Saos-2/Taxol and U-2 OS/Taxol cells. LC3I to LC3II conversion indicates a more pronounced formation of autophagosomes, and the increase of Atg5, Atg7 and Beclin1 is typically suggestive of autophagy onset (3). CAV-1 levels were demonstrated to be reversely correlated with basal autophagy onset in human osteosarcoma cancer cells in the context of Taxol resistance. For the observation of autophagosome formation, it was established that cells stably expressing RFP-LC3 mediated via a lentivirus-associated mechanism. Significantly increased basal autophagy was exhibited by RFP-LC3 puncta in Taxol-resistant cells, with serum starvation used as a positive control for autophagy (Fig. 1C and D; $\mathrm{P}<0.01$ ).

CAV-1 knockdown induces Taxol resistance and autophagy in Saos-2 and U-2 OS cells. To explore the effect of CAV-1 in Taxol resistance and autophagy, a CAV-1 knockdown system was established using lentiviral transduction. To investigate the silencing efficiency of two different shRNA targeting CAV-1, qPCR and western blot analysis were performed in Saos-2 and U-2 OS cells (Fig. 2A and B). Following knockdown of
CAV-1, cells were treated with Taxol $(10 \mathrm{nM})$ and cell viability was examined using an MTT assay kit. As shown in Fig. 2C, knockdown of CAV-1 was induced resistance to Taxol, and when autophagy-related markers were detected, autophagy was significantly enhanced after CAV-1 knockdown $(\mathrm{P}<0.05)$. These results indicate that deficiency of CAV-1 may induce autophagy and Taxol resistance in human osteosarcoma cells.

Overexpression of CAV-1 prevents autophagy and Taxol resistance in Saos-2/Taxol and U-2 OS/Taxol cells. As shown in Fig. 1B, CAV-1 expression was significantly reduced in Taxol-resistant human osteosarcoma cells. To further elucidate the function of CAV-1 in autophagy and Taxol resistance, CAV-1 was overexpressed in Saos-2/Taxol and U-2 OS/Taxol cells (Fig. 3A), and alterations in autophagy and drug resistance were subsequently examined. Autophagy was significantly inhibited following CAV-1 overexpression in Taxol resistance cells (Fig. 3B and $\mathrm{C}$; $\mathrm{P}<0.05$ ). MTT assay was performed to evaluate cell viability, and the viability of Taxol resistant cells was significantly decreased after CAV-1 was overexpressed (Fig. 3D; P<0.05). Notably, cell viability was significantly reduced in the overexpression group even without Taxol treatment (Fig. 3D; $\mathrm{P}<0.05$ ).

Inhibition of autophagy weakens Taxol resistance mediated by $C A V-1$ deficiency. It has previously been reported that various clinical anti-cancer agents, including tamoxifen, cetuximab and imatinib, are able to induce autophagy in cell culture and animal models (5). The present study revealed that autophagy was increased in drug-resistant cells. To assess the role of autophagy in drug resistance, autophagy was inhibited using two different methods; including inhibition of autophagy at the genetic level and administration of an autophagy inhibitor, Baf A1 $(10 \mu \mathrm{M})$. In the present study, Atg5 knockdown was stimulated to hinder autophagy activation (Fig. 4A and B). As shown in Fig. 4C, basal autophagy and autophagy induced by CAV-1 deficiency were blocked by downregulating Atg5. Subsequently, it was demonstrated that Taxol resistance mediated by the loss of CAV-1 in Fig. 2C was markedly decreased after the inhibition of autophagy by downregulating Atg5 (Fig. 4C). When cells were treated with Baf A1 $(10 \mu \mathrm{M})$, drug resistance was declined following autophagy inhibition (Fig. 4D). These results suggest that CAV-1-associated Taxol resistance involves the autophagy pathway.

CAV-1 reduces Taxol resistance and autophagy by JNK signaling. To investigate how CAV-1 reduces Taxol resistance and autophagy, the role of JNKs in this process was analyzed as JNKs have previously been reported to mediate Taxol resistance in ovarian carcinoma cells (19), and JNK1-mediated phosphorylation of B cell lymphoma-2 has an important role in the regulation of autophagy (20). To compare Taxol-resistant cells and their parent cells, the activation of the phosphorylation of JNK and upstream Akt was increased, suggesting an increase in JNK activity (Fig. 5A). The effect of JNK on autophagy and Taxol resistance of osteosarcoma cells was subsequently investigated. Inhibition of JNK activity was achieved by treating cells with a JNK inhibitor (SP600125), which significantly decreased the level 

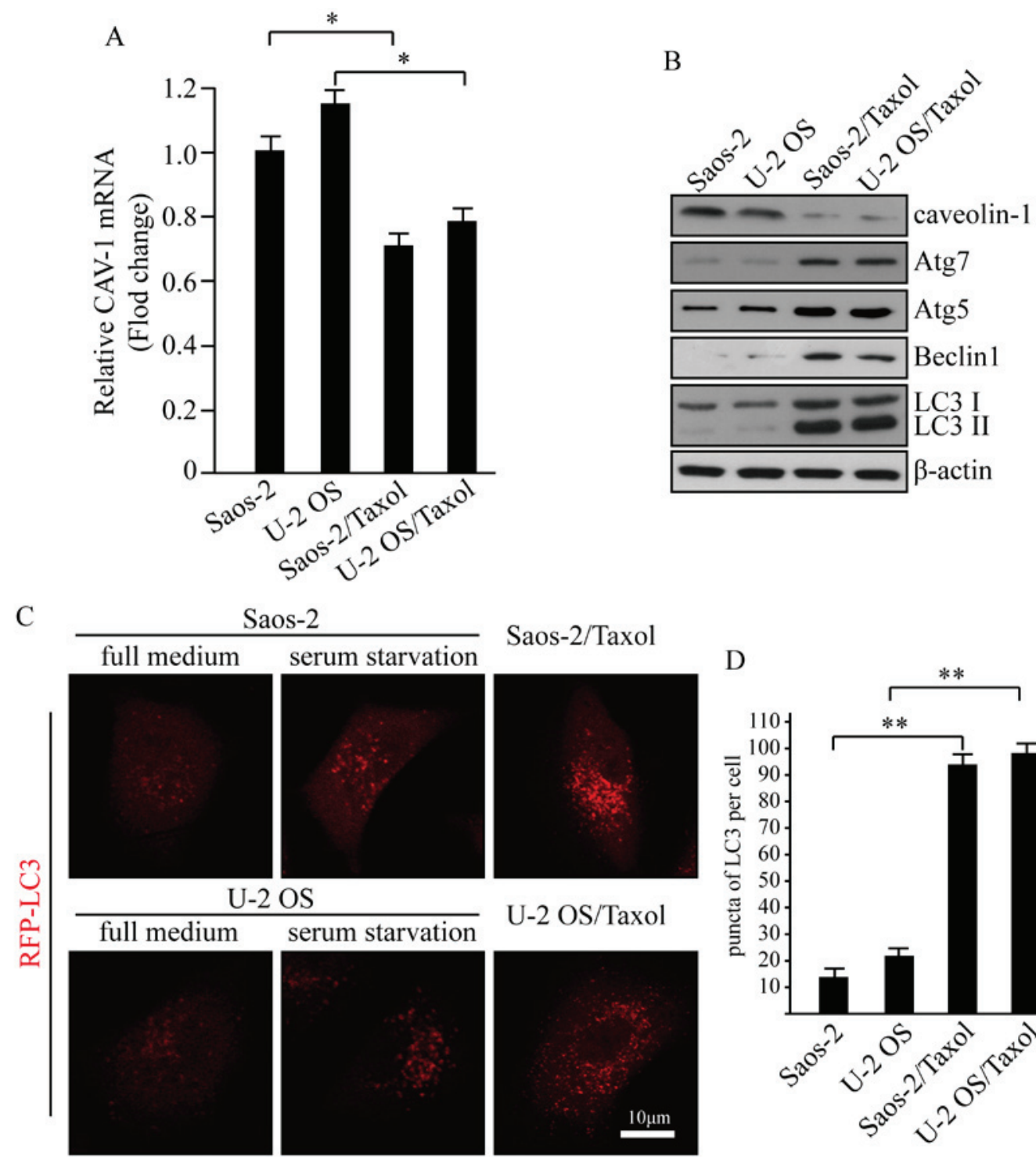

Saos-2/Taxol

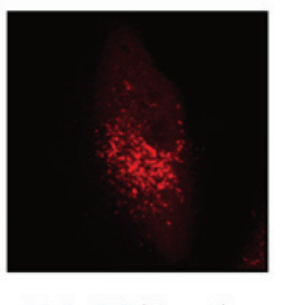

D

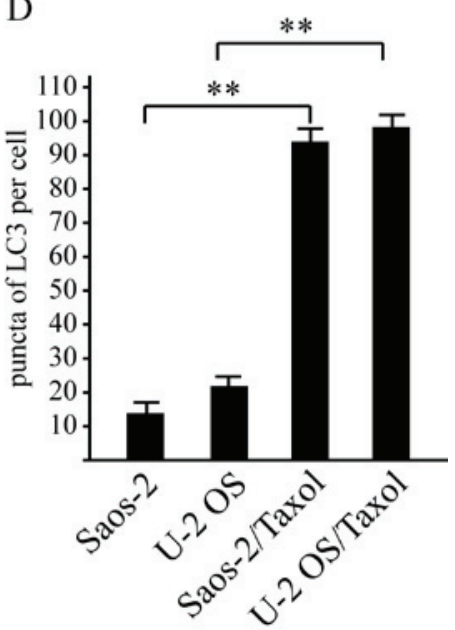

Figure 1. CAV-1 expression and basal autophagy levels are altered in Taxol-resistant human osteosarcoma cells. (A) mRNA transcription levels of CAV-1 are shown for Saos-2, U-2 OS and drug-resistant cells. mRNA levels were normalized to $\beta$-actin and expressed as the fold-change in Saos-2 cells (n=3). (B) Immunoblotting analysis of CAV-1 and canonical autophagy markers in Saos-2/Taxol, U-2 OS /Taxol and their parent cells, with $\beta$-actin as a loading control. (C) Representative confocal microscope images of Saos-2, U-2 OS and drug-resistant cells stably expressing RFP-LC3. Scale bar: $10 \mu \mathrm{m}$. (D) The average number of RFP-LC3 dots per cell was counted in $>15$ fields with at least 200 cells for each group. Data are presented as the mean \pm standard error of the mean of at least three independent experiments. "P $<0.05$; * $\mathrm{P}<0.01$. CAV-1, caveolin-1; OS, osteosarcoma; RFP-LC3, red fluorescent protein conjugated microtubule associated protein 1 light chain 3.

of autophagy and the Taxol resistance of Saos-2/Taxol and U-2 OS cells (Fig. 5B; P<0.05). However, overexpression of CAV-1 abolished the activity of JNK and Akt, and decreased the level of autophagy and Taxol resistance of Saos-2/Taxol and U-2 OS cells (Fig. 5C; $\mathrm{P}<0.05$ ). The expression of a constitutively active JNK rescued the inhibiting effect of CAV-1 on autophagy and Taxol resistance (Fig. 5D). These results indicate that CAV-1 inhibits autophagy and Taxol resistance via JNK signaling. To identify the pathway by which CAV-1 inhibits the activity of JNK, the role of PI3K and Akt in this process was examined. Inhibition of either PI3K or Akt activity using a PI3K inhibitor (LY294002) or Akt inhibitor (MK-2206) decreased JNK activation (Fig. 5E). These results indicate that CAV-1 may inhibit JNK activity via a decrease in PI3K-Akt activity and suggests that
CAV-1 may inhibit autophagy and Taxol resistance via the PI3K-Akt-JNK pathway.

\section{Discussion}

Osteosarcoma is the most prevalent malignant primary sarcoma of bone in children and adolescents, and is a leading cause of cancer-associated mortality in young adults, accounting for 3-4\% of all malignancies in adolescents and $\sim 30 \%$ of malignant bone tumors (1). Standard therapy is typically multimodal, including neoadjuvant chemotherapy and subsequent amputation or limb-sparing reconstructive surgeries, with adjuvant chemotherapy (1). Chemotherapy plays a significant role in improving patient survival and decreasing mortality in cancer patients $(21,22)$; however, 
A

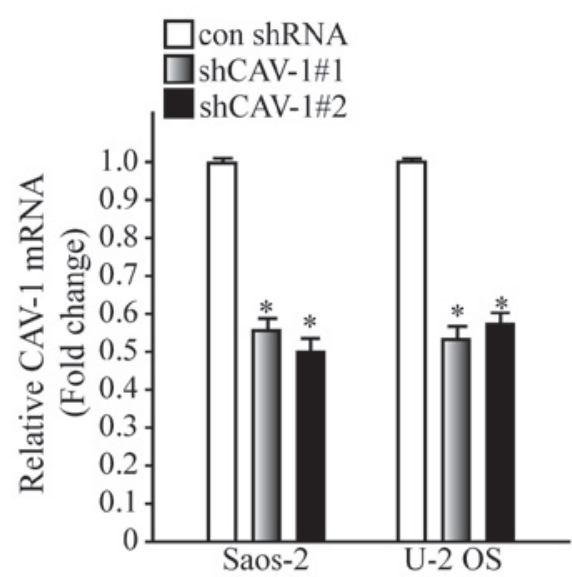

C

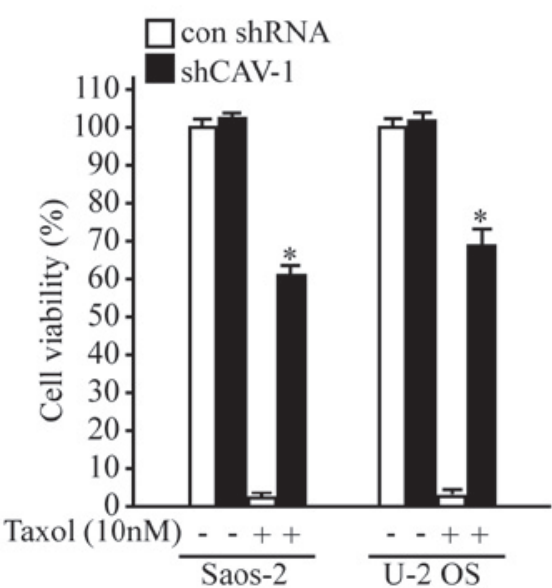

B

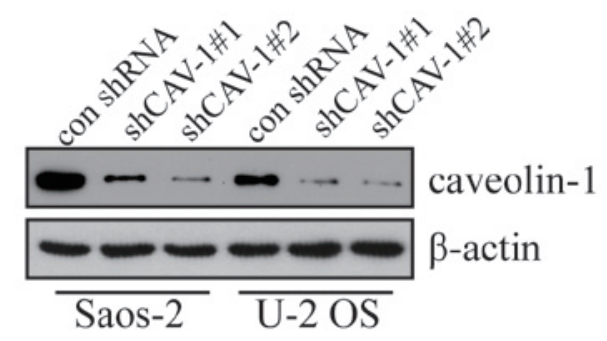

D

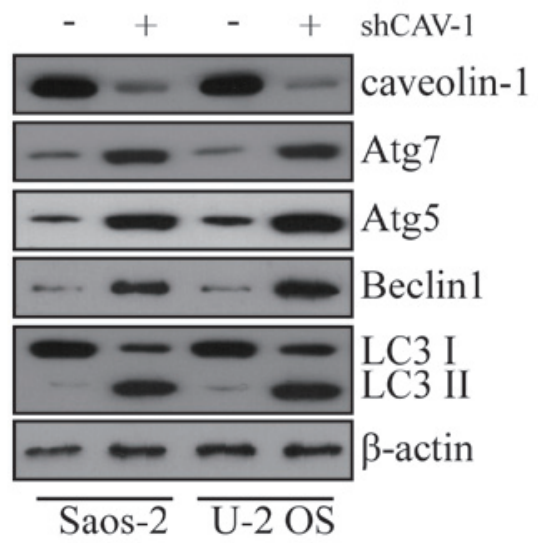

Figure 2. Loss of CAV-1 induces Taxol resistance and autophagy in Saos-2 and U-2 OS cells. (A) Quantitative polymerase chain reaction analysis indicated CAV-1 gene knockdown by shRNA\#1 and 2 as well as control shRNA, $(\mathrm{n}=3)$. (B) Western blot also demonstrated CAV-1 knockdown. (C) Cell viability was detected using an MTT assay after stable knockdown of CAV-1, with Taxol $(10 \mathrm{nM})$ treatment or not $(\mathrm{n}=3)$. Data are presented as the mean \pm standard error of the mean of three independent replicates. (D) Autophagy related genes were detected via western blotting assay after downregulation of CAV-1 gene in Saos-2 and U-2 OS cells. "P<0.05 vs. con shRNA group. CAV-1, caveolin-1; OS, osteosarcoma; sh, short hairpin; con, control.

drug resistance, whether acquired or otherwise, threatens the clinical outcomes and prognoses of patients with cancer.

Genetic alterations are thought to be key factors in the majority of solid tumors. There are numerous molecules correlated with drug resistance, which have not been demonstrated to be causative factors; therefore, these molecular markers are not ideal targets for developing pharmacological agents (5). However, in recent decades, genetic diagnosis and gene therapy have become fast-growing areas of research. Progression in molecular technologies has promoted the elucidation of the mechanisms underlying carcinogenesis, Autophagy mechanisms have captured increasing attention, and autophagy-related genes have become viable prospects for novel potential targets in cancer treatment $(23,24)$.

Autophagy is characterized by the bulk degradation of damaged organelles and misfolding proteins, and is a critical process for cellular homeostasis, differentiation, viability and mammalian development (25). Autophagy has also been demonstrated to cause cell death in certain conditions, working as a tumor suppressing mechanism during the initiation stage of cancer progression (5). Conversely, once the tumor has formed, autophagy assists in the prevention of cell death induced by anticancer therapeutic agents (26).
Therefore, autophagy has two inverse effects in different contexts.

Autophagy has been identified in various tissues, and has been demonstrated to correlate significantly with cancer, cardiomyopathies, neurodegenerative diseases and bacterial infections $(4,5)$. Various proteins are associated with the detection of autophagic activity, including Atg5, Atg7, Beclin1 and the microtubule-associated protein LC3. LC3-I and LC3-II are two cellular forms of LC3 protein; LC3-I is the cytoplasmic form, whereas LC3-II is located in the autophagosomal membrane (7). Therefore, an increase in the conversion of LC3-I to LC3-II is correlated with the extent of autophagosome formation.

To date, the function of CAV-1 in autophagy remains unclear. In the present study, CAV-1 loss was observed in drug-resistant osteosarcoma cells and induced the activation of autophagy. In a previous study, loss of CAV-1 was also reported in various types of malignancies including colon cancer, breast cancer and drug-resistant ovarian carcinoma (27). Furthermore, it has been demonstrated that CAV-1 deletion increased basal autophagy due to an increase in Atg5-Atg12, whereas CAV-1 binding motif mutation broke the association between Atg5 and Atg12 and accelerated autophagy (14). The present 
A

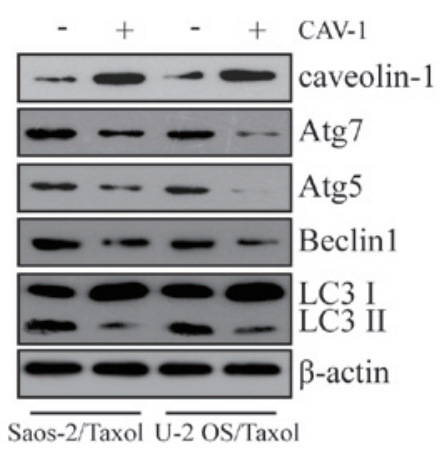

C

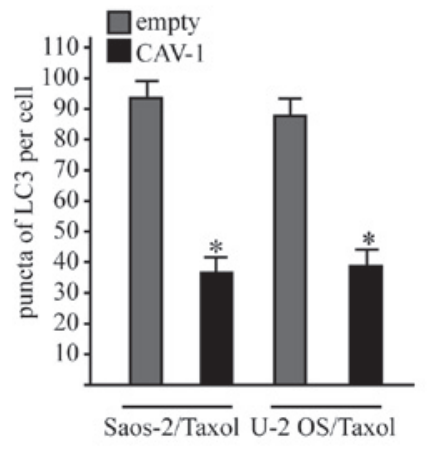

B

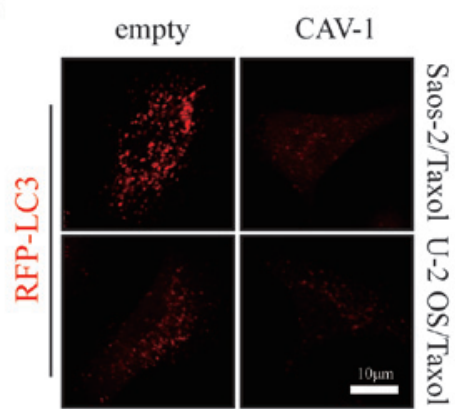

D

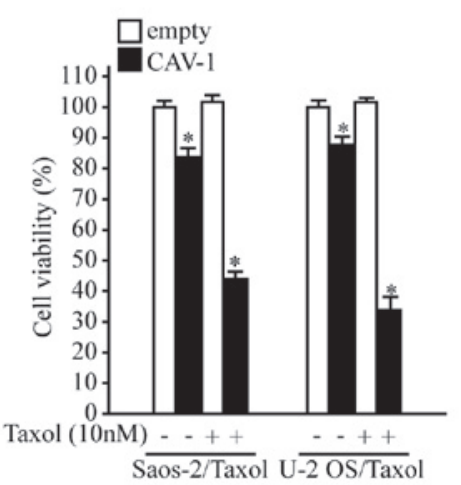

Figure 3. Overexpression of CAV-1 prevents autophagy and Taxol resistance in Saos-2/Taxol and U-2 OS/Taxol cells. (A) Western blot analysis of CAV-1 and autophagy markers in Saos-2/Taxol and U-2 OS/Taxol cells stably overexpressing CAV-1. (B) Representative confocal microscope images of Saos-2/Taxol and U-2 OS/Taxol cells stably expressing RFP-LC3, with overexpression of CAV-1 and empty vector control as illustrated. Scale bar: $10 \mu \mathrm{m}$. (C) The average number of RFP-LC3 dots per cell was counted in $>15$ fields with at least 200 cells for each group. (D) Cell viability was detected through MTT assay after stable overexpression of CAV-1, with Taxol $(10 \mathrm{nM})$ treatment or not $(\mathrm{n}=3)$. Data are presented as the mean \pm standard error of the mean of three independent replicates. "P<0.05 vs. empty (vector) group. OS, osteosarcoma; RFP-LC3, red fluorescent protein conjugated microtubule associated protein 1 light chain 3.

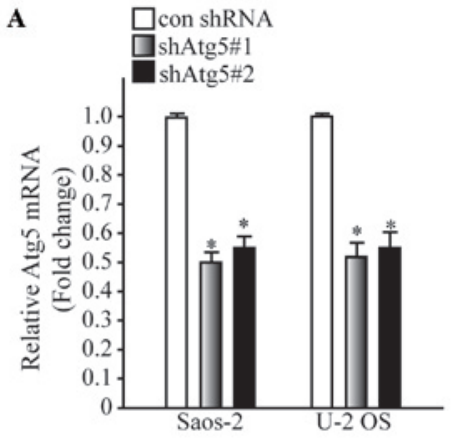

D

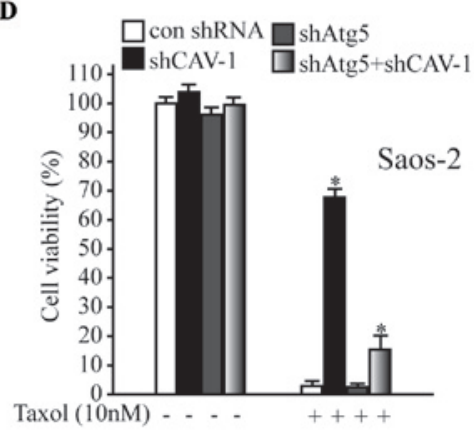

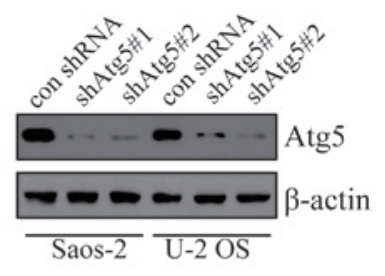

C

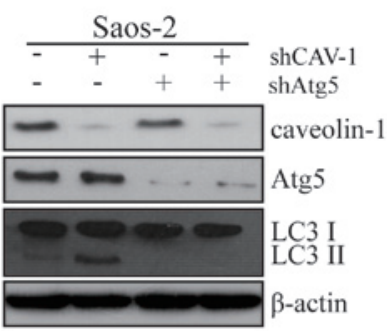

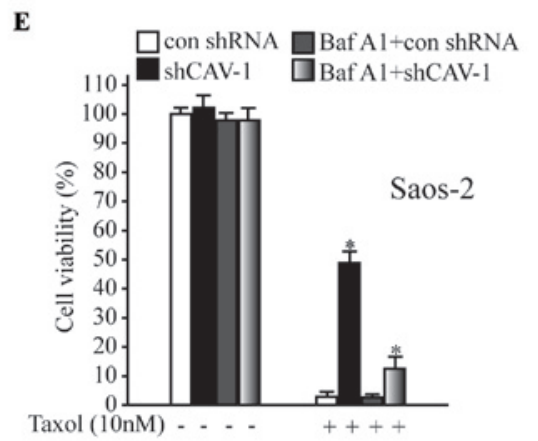

Figure 4. Inhibition of autophagy impairs Taxol resistance mediated by CAV-1 deficiency. (A) Qualitative polymerase chain reaction analysis indicated Atg5 gene knockdown effect by two different shRNA, as well as control shRNA (n=3). (B) Atg5 knockdown effect was indicated using western blotting analysis. (C) Western blotting analysis of autophagy induced by downregulation of CAV-1 with Atg5 knockdown lentivirus treatment or not. (D) Cell viability was examined using an MTT kit after stable downregulation of CAV-1, Atg5 or both (n=3). (E) Cell viability was examined through an MTT assay after stable downregulation of CAV-1, or treated with Baf A1 $(10 \mu \mathrm{M})$ for $4 \mathrm{~h}(\mathrm{n}=3)$. Data are presented as the mean \pm standard error of the mean of three independent replicates. "P<0.05 vs. con shRNA group. CAV-1, caveolin-1; Atg5, autophagy-related 5; OS, osteosarcoma; sh, short hairpin; con, control. 


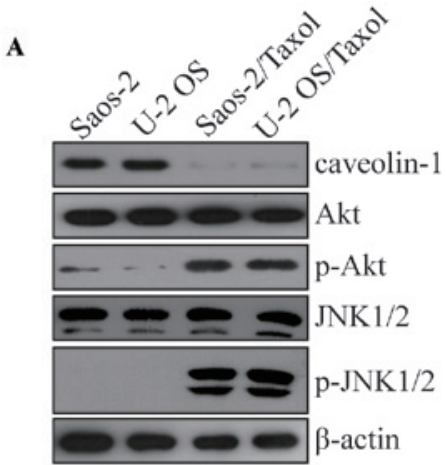

B

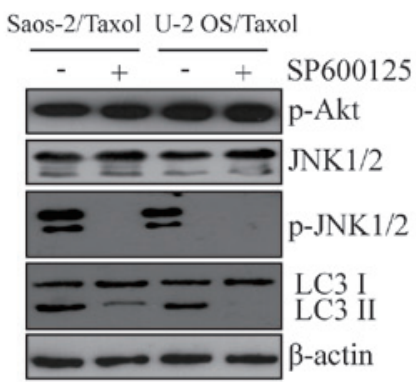

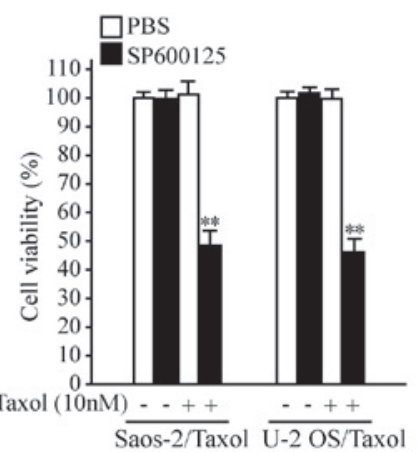

C
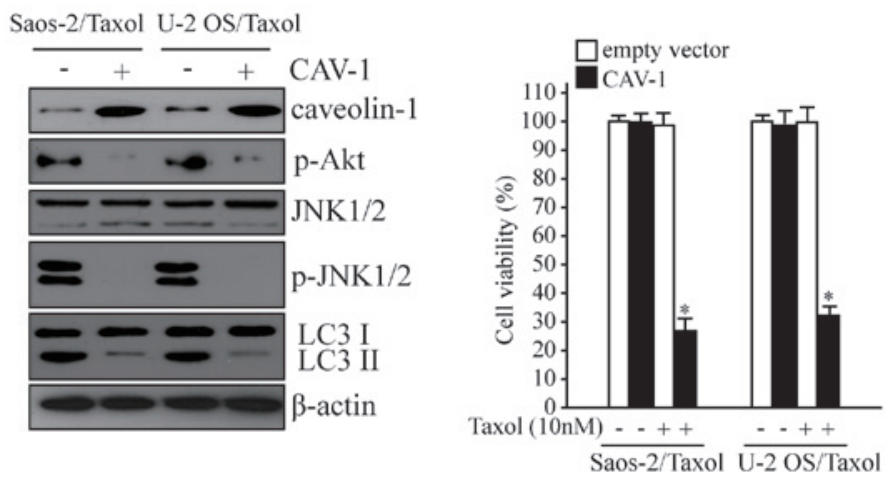

D
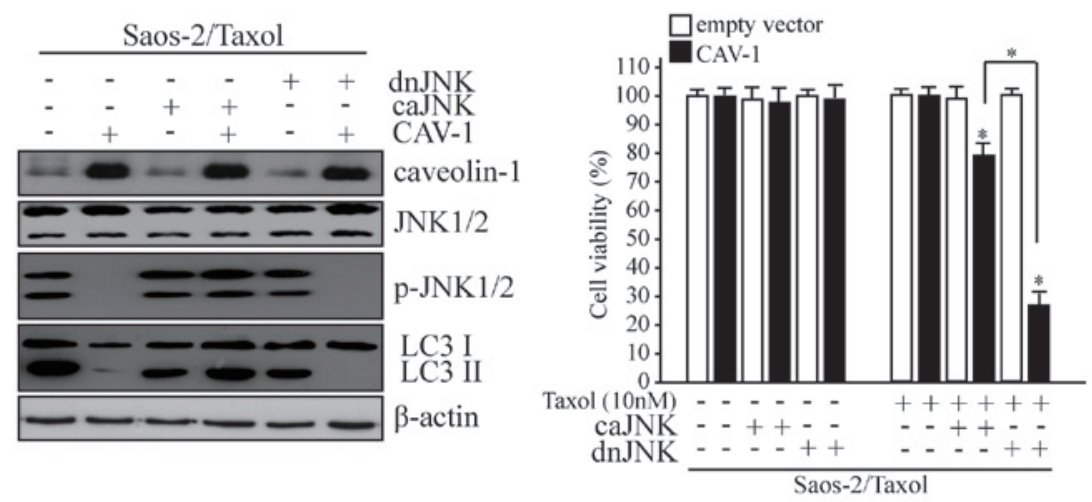

$\mathbf{E}$

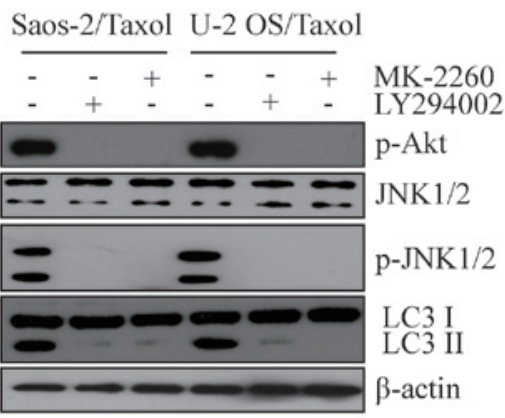

$\mathbf{F}$

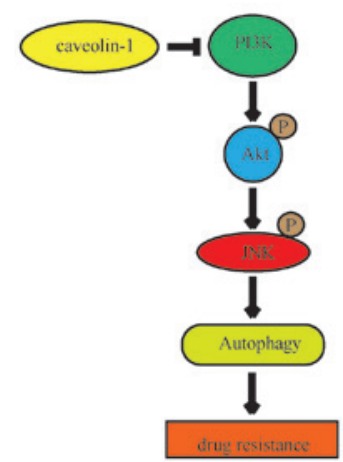

Figure 5. CAV-1 reduces Taxol resistance and autophagy by the JNK signaling. (A) Detection of JNK activity and upstream Akt activity in parental cells and Taxol-resistant cells. (B) Effects of JNK inhibition on autophagy and Taxol resistance. Saos-2/Taxol and U-2 OS cells were treated with $10 \mu$ M SP600125 for $1 \mathrm{~h}$ before analysis. (C) Effects of CAV-1 overexpression on autophagy and Taxol resistance. Saos-2/Taxol and U-2 OS cells were transfected with a CAV-1 or control vector for $48 \mathrm{~h}$ prior to Taxol exposure. (D) Rescue effects of constitutively active JNK on autophagy and Taxol resistance. Saos-2/Taxol cells were transfected with a caJNK or dnJNK after CAV-1 overexpression for $48 \mathrm{~h}$ prior to analysis. Data are presented as the mean \pm standard error of the mean of three independent replicates. " $\mathrm{P}<0.05$ vs. empty vector group; ${ }^{* *} \mathrm{P}<0.05$ vs. PBS group. (E) Effects of LY294002 or MK-2206 on the phosphorylation of JNK. Saos-2/Taxol and U-2 OS cells were treated with $10 \mu \mathrm{M} \mathrm{LY294002} \mathrm{or} 10 \mu \mathrm{M}$ MK-2206 for $1 \mathrm{~h}$ before western blot analysis. (F) A suggested model for how CAV-1 reduces Taxol resistance by inhibiting autophagy. CAV-1, caveolin-1; OS, osteosarcoma; JNK, Janis kinase; caJNK, constitutively active JNK; dnJNK, dominant-negative JNK. 
study also provided evidence that CAV-1 loss contributed to autophagy and the Taxol resistance of Saos-2 and U-2 OS cells via the PI3K-Akt-JNK pathway; whereas the overexpression of CAV-1 inhibited autophagy and declined Saos-2/Taxol and U-2 OS/Taxol resistance to Taxol. Interrupting autophagy genetically (via the knockdown of Atg5) or with an autophagy inhibitor (Baf A1) impaired the drug resistance induced by CAV-1 deficiency. Previous studies have similarly reported that CAV-1 deficiency was an independent factor for the poor prognosis of colorectal cancer individuals, demonstrating that loss of CAV-1 may assist drug resistance and cancer metastasis $(15,16)$.

In conclusion, the results of the present study demonstrated a novel function of CAV-1 in human osteosarcoma cells, indicating that attenuating autophagy may be a promising potential therapeutic approach. In the future, detection of CAV-1 may be a good indicator with which to evaluate the treatment and prognosis of osteosarcoma.

\section{Acknowledgements}

This work was supported by Science Computing and Intelligent Information processing of GuangXi Higher Education Key Laboratory (grant no. GXSCIIP201502).

\section{References}

1. Isakoff MS, Bielack SS, Meltzer P and Gorlick R: Osteosarcoma: Current treatment and a collaborative pathway to success. J Clin Oncol 33: 3029-3035, 2015.

2. Galluzzi L, Pietrocola F, Levine B and Kroemer G: Metabolic control of autophagy. Cell 159: 1263-1276, 2014.

3. Klionsky DJ and Emr SD: Autophagy as a regulated pathway of cellular degradation. Science 290: 1717-1721, 2000.

4. Choi AM, Ryter SW and Levine B: Autophagy in human health and disease. N Engl J Med 368: 651-662, 2013.

5. Levine B and Kroemer G: Autophagy in the pathogenesis of disease. Cell 132: 27-42, 2008.

6. Cicchini M, Chakrabarti R, Kongara S, Price S, Nahar R, Lozy F, Zhong H, Vazquez A, Kang Y and Karantza V: Autophagy regulator BECN1 suppresses mammary tumorigenesis driven by WNT1 activation and following parity. Autophagy 10: 2036-2052, 2014.

7. Zhang F, Kumano M, Beraldi E, Fazli L, Du C, Moore S, Sorensen P, Zoubeidi A and Gleave ME: Clusterin facilitates stress-induced lipidation of LC3 and autophagosome biogenesis to enhance cancer cell survival. Nat Commun 5: 5775, 2014

8. Wang L, Zhang H, Sun M, Yin Z and Qian J: High mobility group box 1-mediated autophagy promotes neuroblastoma cell chemoresistance. Oncol Rep 34: 2969-2976, 2015.

9. Chen S, Zhu X, Qiao H, Ye M, Lai X, Yu S, Ding L, Wen A and Zhang J: Protective autophagy promotes the resistance of HER2-positive breast cancer cells to lapatinib. Tumour Biol 37: 2321-2331, 2016

10. Giuliano S, Cormerais Y, Dufies M, Grépin R, Colosetti P, Belaid A, Parola J, Martin A, Lacas-Gervais S, Mazure NM, et al: Resistance to sunitinib in renal clear cell carcinoma results from sequestration in lysosomes and inhibition of the autophagic flux. Autophagy 11: 1891-1904, 2015.
11. Crystal AS, Shaw AT, Sequist LV, Friboulet L, Niederst MJ, Lockerman EL, Frias RL, Gainor JF, Amzallag A, Greninger P, et al: Patient-derived models of acquired resistance can identify effective drug combinations for cancer. Science 346: 1480-1486, 2014.

12. Wang Z, Wang N, Li W, Liu P, Chen Q, Situ H, Zhong S, Guo L, Lin Y, Shen J and Chen J: Caveolin-1 mediates chemoresistance in breast cancer stem cells via $\beta$-catenin/ABCG2 signaling pathway. Carcinogenesis 35: 2346-2356, 2014.

13. Shi Y, Tan SH, Ng S, Zhou J, Yang ND, Koo GB, McMahon KA, Parton RG, Hill MM, Del Pozo MA, et al: Critical role of CAV1/caveolin-1 in cell stress responses in human breast cancer cells via modulation of lysosomal function and autophagy. Autophagy 11: 769-784, 2015.

14. Shiroto T, Romero N, Sugiyama T, Sartoretto JL, Kalwa H, Yan Z, Shimokawa H and Michel T: Caveolin-1 is a critical determinant of autophagy, metabolic switching, and oxidative stress in vascular endothelium. PLoS One 9: e87871, 2014

15. Wu KN, Queenan M, Brody JR, Potoczek M, Sotgia F, Lisanti MP and Witkiewicz AK: Loss of stromal caveolin-1 expression in malignant melanoma metastases predicts poor survival. Cell Cycle 10: 4250-4255, 2011.

16. Zhao Z, Han FH, Yang SB, Hua LX, Wu JH and Zhan WH: Loss of stromal caveolin-1 expression in colorectal cancer predicts poor survival. World J Gastroenterol 21: 1140-1147, 2015.

17. Livak KJ and Schmittgen TD: Analysis of relative gene expression data using real-time quantitative PCR and the 2(-Delta Delta C (T)) Method. Methods 25: 402-408, 2001.

18. Pu J, Schindler C, Jia R, Jarnik M, Backlund P and Bonifacino JS: BORC, a multisubunit complex that regulates lysosome positioning. Dev Cell 33: 176-188, 2015.

19. Sun NK, Huang SL, Lu HP, Chang TC and Chao CC: Integrative transcriptomics-based identification of cryptic drivers of taxol-resistance genes in ovarian carcinoma cells: Analysis of the androgen receptor. Oncotarget 6: 27065-27082, 2015.

20. Wei Y, Pattingre S, Sinha S, Bassik M and Levine B: JNK1-mediated phosphorylation of $\mathrm{Bcl}-2$ regulates starvation-induced autophagy. Mol Cell 30: 678-688, 2008.

21. Siegel R, DeSantis C, Virgo K, Stein K, Mariotto A, Smith T, Cooper D, Gansler T, Lerro C, Fedewa S, et al: Cancer treatment and survivorship statistics, 2012. CA Cancer J Clin 62: 220-241, 2012.

22. Li Y, Dang TA, Shen J, Hicks J, Chintagumpala M, Lau CC and Man TK: Plasma proteome predicts chemotherapy response in osteosarcoma patients. Oncol Rep 25: 303-314, 2011.

23. Shibutani ST, Saitoh T, Nowag H, Münz C and Yoshimori T: Autophagy and autophagy-related proteins in the immune system. Nat Immunol 16: 1014-1024, 2015.

24. Bao XH, Naomoto Y, Hao HF, Watanabe N, Sakurama K, Noma K, Motoki T, Tomono Y, Fukazawa T, Shirakawa Y, et al: Autophagy: Can it become a potential therapeutic target? Int J Mol Med 25: 493-503, 2010.

25. Axe EL, Walker SA, Manifava M, Chandra P, Roderick HL, Habermann A, Griffiths G and Ktistakis NT: Autophagosome formation from membrane compartments enriched in phosphatidylinositol 3-phosphate and dynamically connected to the endoplasmic reticulum. J Cell Biol 182: 685-701, 2008.

26. Levine B: Cell biology: Autophagy and cancer. Nature 446: 745-747, 2007.

27. Williams TM and Lisanti MP: Caveolin-1 in oncogenic transformation, cancer, and metastasis. Am J Physiol Cell Physiol 288: C494-C506, 2005. 